\title{
Die deutschen Arbeitsmarktreformen: Nicht perfekt, aber unter dem Strich positiv
}

\section{WARUM KAM ES ZU DEN REFORMEN?}

Die Agenda 2010 und die Hartzreformen müssen vor dem Hintergrund der deutschen Wirtschaftsgeschichte seit Ende der Vollbeschäftigungsphase Mitte der 1970er Jahre gesehen werden. Seit dieser Zeit befand sich die Arbeitsmarktentwicklung in Deutschland auf einer schiefen Ebene. Jede der schweren Rezessionen (die erste und zweite Ölpreiskrise 1974/75 bzw. 1981, die Wiedervereinigungskrise 2003/2004 und die Krise nach dem Zusammenbruch der Dotcom-Blase 2003/2004) hat in Deutschland dauerhaft Wunden hinterlassen. Die ergriffenen Politikmaßnahmen - seien es die verschiedenen Ansätze von Beschäftigungs- und Arbeitsmarktpolitik oder Maßnahmen zur Angebotsverknappungerwiesen sich als ungeeignet, den Kern der Arbeitslosigkeit nachhaltig zu reduzieren und den Arbeitsmarkt zu einem Gleichgewicht zurückzuführen.

Am Beginn des neuen Jahrtausends hatte die Sockelarbeitslosigkeit schließlich eine Höhe erreicht, die nicht mehr tragbar erschien. Die Arbeitslosigkeit hatte sich verhärtet, der Anteil der Langzeitarbeitslosen war auf sehr hohe Werte gestiegen. Die internationale Wirtschaftspresse bezeichnete damals Deutschland wohl nicht ganz ohne Grund als „kranken Mann Europas“.

Auffällig war eine Besonderheit in der Dynamik der deutschen Arbeitsmarktentwicklung seit Mitte der 1970er Jahre. Während in anderen Ländern (etwa den USA) die im Abschwung entstandene Arbeitslosigkeit im Aufschwung vergleichsweise schnell wieder abgebaut wurde - Anstieg und Abfall sich also mehr oder weniger symmetrisch vollzogen-, war für Deutschland eine starke Asymmetrie kennzeichnend: Einem massiven Aufbau der Arbeitslosigkeit in der Krise folgte ein verhaltener und kraftloser Abbau im Aufschwung. In der Konsequenz stieg die Sockelarbeitslosigkeit nach jeder Krise. Dieser Sachverhalt ist häufig beschrieben und mit dem Begriff „Hysteresis“ (Zurückbleibeeffekt) belegt worden; die Folgen der Krise bildeten sich nicht wieder zurück, als die Auslöser verschwanden.
Auch vor dem Hintergrund der unerwartet hohen und andauernden Belastungen durch die deutsche Wiedervereinigung war diese Situation weder finanziell noch gesellschaftlich auf Dauer akzeptabel. Mit der Agenda 2010 reagierte die Regierung Schröder auf diese Erkenntnis. Der Kernsatz aus der Regierungserklärung von 2003 lautet: „Entweder wir modernisieren, und zwar als soziale Marktwirtschaft, oder wir werden modernisiert, und zwar von den ungebremsten Kräften des Marktes, die das Soziale beiseite drängen würden“. Einen Eindruck von dem, was das Letztere bedeutet, bekommt man, wenn man an die momentane Situation in Griechenland denkt.

\section{INSTITUTIONEN UND VERHALTEN}

Die Gretchenfrage lautet: Wie kommt es im internationalen Vergleich zu den augenfälligen Unterschieden im dynamischen Verhalten des Arbeitsmarktes? Als Ursache kommen in erster Linie Differenzen in institutionellen Regelungen in Betracht, die auf das Verhalten der Akteure am Arbeitsmarkt (Erwerbspersonen ebenso wie Firmen) zurückwirken. Dass es solche Wirkungen gibt, steht außer Frage. Dies belegen Phänomene wie z. B. die Häufung der Beendigung von Arbeitslosigkeit durch Wiederaufnahme einer Beschäftigung kurz vor dem Auslaufen von Unterstützungsleistungen oder die auffällig erhöhten Zugänge in Arbeitslosigkeit, kurz bevor eine Verkürzung der Bezugsdauer in Kraft trat. ${ }^{1}$ Diese Sachverhalte sind kein Zufall, sondern Konsequenz der Tatsache, dass Arbeitnehmer und Firmen ihr Verhalten tendenziell an die jeweiligen Gegebenheiten in einer für sie bestmöglichen Weise anpassen. Das ist ebenso menschlich wie verständlich. Man muss die Augen schon sehr stark verschließen, wenn man diese offensichtlichen Phänomene ignorieren möchte, um ein idealisiertes Menschenbild aufrecht zu erhalten. ${ }^{2}$ Die Argumentation, dass die Hartzreformen keine Wirkungen zeigen konnten, weil sie vom falschen Menschenbild ausgingen (Niemeier in diesem Heft), überzeugt deshalb nicht. Etwas fühle ich mich an Christian
Morgensterns Palmström erinnert: „Weil, so schließt er messerscharf, nicht sein kann, was nicht sein darf", oder an die Anekdote von den scholastischen Mönchen im Mittelalter, die ausgiebig darüber diskutierten, ob Milch gefrieren könne. Man versuchte das Ergebnis aus allgemeinen Prinzipien abzuleiten, anstatt eine Schüssel in die Kälte zu stellen, um zu sehen was passiert.

\section{SCHWACHSTELLEN DES ALTEN SYSTEMS}

Das Sozialhilfemodell vor den Hartzreformen wird heute häufig idealisiert dargestellt. Es hatte jedoch entscheidende Schwächen. Die unklare Zuweisung der Verantwortlichkeiten auf die verschiedenen Träger führte zu inakzeptablen finanziellen Verschiebebahnhöfen zwischen Grundsicherung und Arbeitslosenversicherung. Die Gewährung von Leistungen nach dem Einzelfall zog nicht nur einen erheblichen bürokratischen Aufwand nach sich, sondern auch erhebliche Ungerechtigkeiten, da bedürftige Personen aus Unkenntnis oder Scham die ihnen zustehenden Leistungen oft nicht in Anspruch nahmen. Zudem wurden in der Sozialhilfe viele erwerbsfähige Personen versteckt, obwohl sie arbeitslos waren. Systematische Unterstützung bei der Arbeitssuche gab es in den Sozialämtern zumeist nicht. Dem System fehlte demnach

\footnotetext{
1 Vgl. Dlugosz, S./Stephan, G./Wilke, R. A. (2009): Fixing the leak: Unemployment incidence before and after the 2006 reform of unemployment benefits in Germany, IAB Discussion Paper 25, Nürnberg. 2 Dies heißt nicht, dass sich bestimmte Individuen nicht dem Idealbild entsprechend verhalten können.
}

Joachim Möller, Prof. Dr. Dr. h. c., Volkswirt Direktor des Instituts für Arbeitsmarkt- und Berufsforschung (IAB) der Bundesagentur für Arbeit (BA) sowie Lehrstuhlinhaber und Professor für Volkswirtschaftslehre an der Universität Regensburg. Arbeitsschwerpunkte: Empirische Makroökonomie und Regionalökonomie. e-mail: joachim.moeller@iab.de 


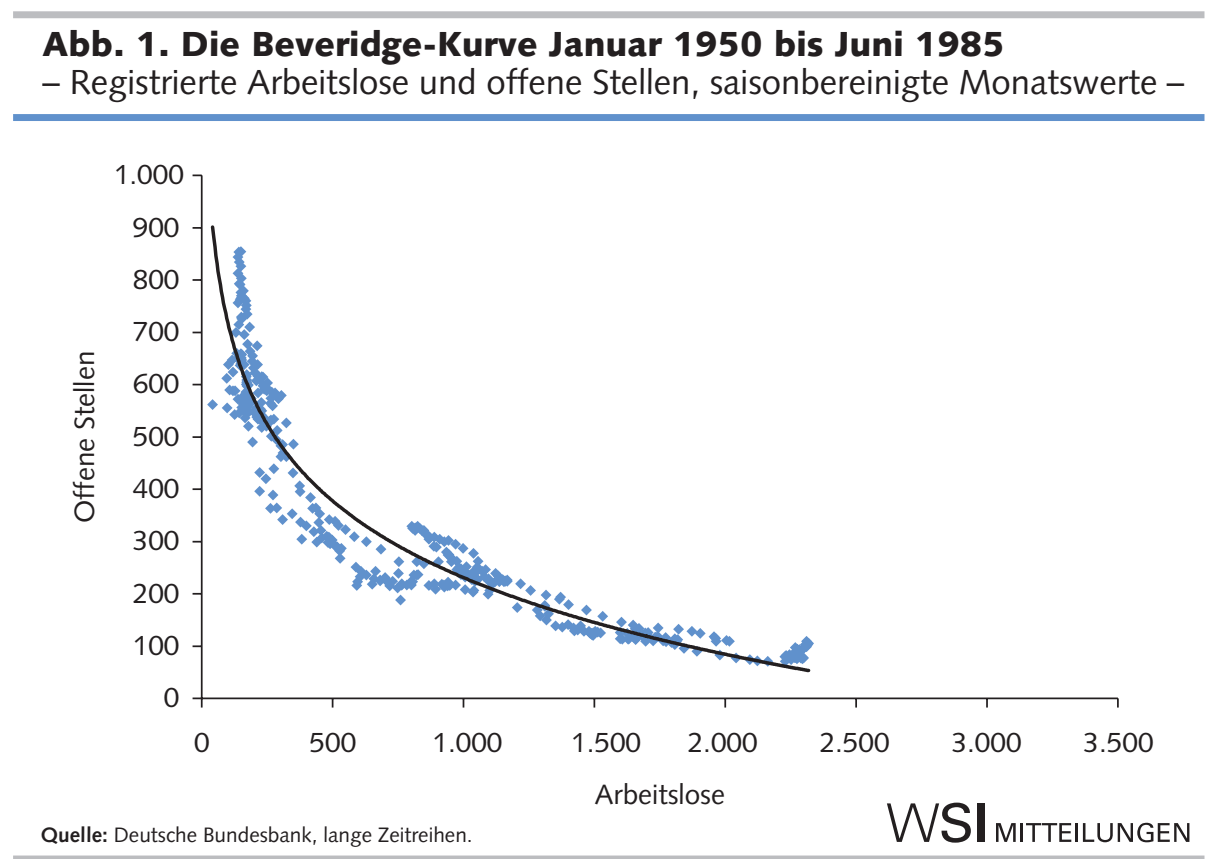

nicht nur die finanzielle Nachhaltigkeit, es führte auch zu deutlich sichtbaren gesellschaftlichen Erosionserscheinungen. Mit dem neuen Ansatz, durch „Fordern und Fördern" bedürftige Personen wieder in Erwerbsarbeit zu integrieren, sollte hier wirksam entgegen gesteuert werden.

\section{VERHÄRTUNG VON ARBEITS- LOSIGKEIT}

Arbeitslosigkeit ist ein schlimmes Übel, insbesondere dann, wenn sie sich verhärtet. Je länger sie andauert, desto schwieriger wird es, sie zu verlassen. Zwei Mechanismen sind dafür verantwortlich: Kompetenzverlust und Stigmatisierung. Langzeitarbeitslose verlieren ihre fachlichen Kenntnisse, aber auch einige „weiche Kompetenzen“, je länger sie aus dem Erwerbsleben heraus sind. Potenzielle Arbeitgeber unterstellen dies und ziehen Kurzzeit- den Langzeitarbeitslosen vor. Es beginnt ein Teufelskreis, der für die Betroffenen häufig mit Frustration, Resignation, materiellem Abstieg und sozialem Ausschluss endet.

Wie kann man diesen Teufelskreis verhindern? Natürlich werden sich Personen, die arbeitslos geworden sind, in aller Regel umgehend auf die Suche nach einem neuen Arbeitsplatz machen. Der überwiegenden Zahl gelingt dies auch in kurzer Zeit, die große Masse der Arbeitslosigkeitsfälle dauert ja nur wenige Monate. Verschiedene Faktoren spielen eine Rolle, ob eine rasche Beendigung der Arbeitslosigkeit gelingt. Wichtige
Faktoren sind die Zahl der verfügbaren Jobs, die Transparenz des Arbeitsmarktes, die Effizienz der Vermittlung, Umfang und Inhalt der Ausbildung. Nicht unbedeutend sind darüber hinaus aber auch die Intensität, mit der der oder die Einzelne sucht, sowie die Bereitschaft, einen Arbeitsplatz anzunehmen, der nicht den Idealvorstellungen entspricht. Sicherlich gibt es Personen, die trotz intensiver Bemühungen und Konzessionsbereitschaft langfristig arbeitslos werden, weil die Jobs fehlen oder die Qualifikationen nicht passen. Ebenso wenig ist aber von der der Aussicht auf eine vergleichsweise lange Lohnersatzleistung nach einem Jobverlust in vermeintlicher Sicherheit wiegen und $\mathrm{zu}$ geringe Integrationsbemühungen aufbringen. In diesen Fällen könnte sich eine (sozialpolitisch gut gemeinte) lange Bezugsdauer von Arbeitslosengeld I als „süßes Gift“ für die Betroffenen entpuppen.

Natürlich besteht an dieser Stelle ein Abwägungsproblem: Auf die richtige Mischung von Fordern und Fördern kommt es an. Wenn durch Fordern ohne Fördern Personen bei Jobverlust gezwungen sind, die nächstbeste freie Stelle anzunehmen, kommt es wegen fehlender Passgenauigkeit zwischen den Kompetenzen des Arbeitssuchenden und den Anforderungen der Stelle zu ökonomischen Effizienzverlusten. Bei Fördern ohne Fordern drohen hingegen Maßnahmekarrieren, die die Betroffenen keineswegs näher an eine Integration in den ersten Arbeitsmarkt heranbringen. Beides ist volkswirtschaftlich nicht sinnvoll. Hand zu weisen, dass sich andere wegen
Die Grundidee der Aktivierung ist es, für jede Person individuell die richtige Mischung von Fördern und Fordern zusammenzustellen, um eine möglichst rasche und dauerhafte Integration in Beschäftigung zu erreichen. Dies ist sicher noch nicht in allen Fällen in der Umsetzung tatsächlich gelungen. Es ist aber keineswegs so, dass hinter dieser Idee die einseitige Vorstellung steckt, die Arbeitslosigkeit sei ausschließlich durch den Arbeitsunwillen der Arbeitslosen verursacht, wie Niemeier (in diesem Heft) in einer sehr engen Interpretation der gesetzlichen Intention vermutet.

\section{MAKROÖKONOMISCHE ARGUMENTE}

Tatsache ist: Im Aufschwung, der von 2005 bis Mitte 2008 dauerte, ist die Arbeitslosigkeit von 5 auf 3 Mio gesunken. Bemerkenswert sind der erhebliche Rückgang der Langzeitarbeitslosigkeit und nicht zuletzt der Aufbau ungewöhnlich vieler Beschäftigungsverhältnisse im Aufschwung, die der Sozialversicherungspflicht unterliegen. Das sind unzweifelhaft Erfolge. Zugleich ist die Zahl der erwerbsfähigen Hilfebedürftigen im SGB II um etwa eine halbe Million zurückgegangen, auch dies ein Hoffnungszeichen, obwohl sich die Väter der Hartzreformen an dieser Stelle sicher mehr versprochen hatten.

Niemeier (in diesem Heft) argumentiert, die genannten Verbesserungen seien auf Konjunkturwirkungen zurückzuführen. Auf der einen Seite ist dies insofern richtig, als ein starker Abbau der Arbeitslosigkeit in der Regel nur in einer guten Konjunkturphase gelingen kann (Okun'sches Gesetz). Auf der anderen Seite ist es aber falsch, die besonderen Eigenschaften des Aufschwungs allein auf äußere Faktoren („Weltkonjunktur") zu schieben. Stärke, Umfang und insbesondere die hohe Beschäftigungsintensität des Aufschwungs sind nicht unabhängig von den Bedingungen, unter denen er stattgefunden hat. ${ }^{3}$ Wohl kann man die weltweite Nachfrageentwicklung aus Sicht des Inlands als exogen ansehen. Welches Land in welchem Umfang davon profitiert

\footnotetext{
3 Ebenso wie das deutsche Jobwunder in der durch die Finanzkrise ausgelösten Weltrezession wiederum nicht unabhängig von den hier vorherrschenden institutionellen Bedingungen erklärt werden kann, vgl. Möller, J. (2010): The German labor market response in the world recession - demystifying a miracle, in: Zeitschrift für ArbeitsmarktForschung 4, S. 325-336.
} 
und wie viel Beschäftigung durch die zusätzlichen Aufträge generiert wird, hängt nicht nur von der Produktpalette des jeweiligen Landes ab, sondern auch von Kostenund Produktivitätsfaktoren sowie davon, wie gut der Arbeitsmarkt funktioniert. Aus meiner Sicht sprechen alle vorliegenden Indizien dafür, dass es günstige Reformwirkungen gegeben hat.

\section{ZUR INTERPRETATION DER BEVERIDGE-KURVE}

Der Zusammenhang zwischen Arbeitslosen und offenen Stellen lässt sich idealtypisch als fallende Kurve darstellen, die konvex zum Ursprung verläuft. Diese Form der Beveridge-Kurve ist empirisch gut nachgewiesen, auch wenn sich bei der Anwendung auf reale Daten Probleme ergeben. Eines besteht in der statistischen Erfassung von Arbeitslosen und offenen Stellen. Letztere werden nur $\mathrm{zu}$ einem Bruchteil registriert oder auf andere Weise erfasst - und auch die Arbeitslosenstatistik ist ja aus bekannten Gründen nicht unumstritten, weil es einen diskretionären Spielraum bei der Festlegung der Erfassungskriterien gibt. In ihrer einfachen Form vernachlässigt die Beveridge-Kurve außerdem Anpassungsvorgänge bzw. -verzögerungen am Arbeitsmarkt.

Wegen der genannten Probleme darf man keineswegs den Fehler machen, den Lehrbuchfall direkt auf die Realität zu übertragen. Im Lehrbuch entspricht (bei gleichem Maßstab auf beiden Achsen) die Winkelhalbierende einem „Gleichgewicht“ in dem Sinn, dass rechnerisch auf jeden Arbeitslosen eine offene Stelle kommt. Bei einer empirischen Beveridge-Kurve ist aber ein solches Vorgehen wegen der statistischen Erfassungsprobleme und nicht berücksichtigten Anpassungsvorgänge nicht sinnvoll. Trotzdem enthält die empirische Beveridge-Kurve wichtige Informationen, die Rückschlüsse auf die Funktionsweise des Arbeitsmarktes erlauben.

Abbildung 1 zeigt den Zusammenhang zwischen registrierten Arbeitslosen und gemeldeten offenen Stellen in Form saisonbereinigter Monatsdaten im Zeitraum Januar 1950 bis Mitte 1985 für Westdeutschland. Die Daten bestätigen den theoretischen Zusammenhang einer negativen Relation, die in diesem Zeitraum (trotz einiger kurzfristiger Abweichungen) bemerkenswert stabil ist. Abbildung 2 erweitert den Beobachtungszeitraum bis

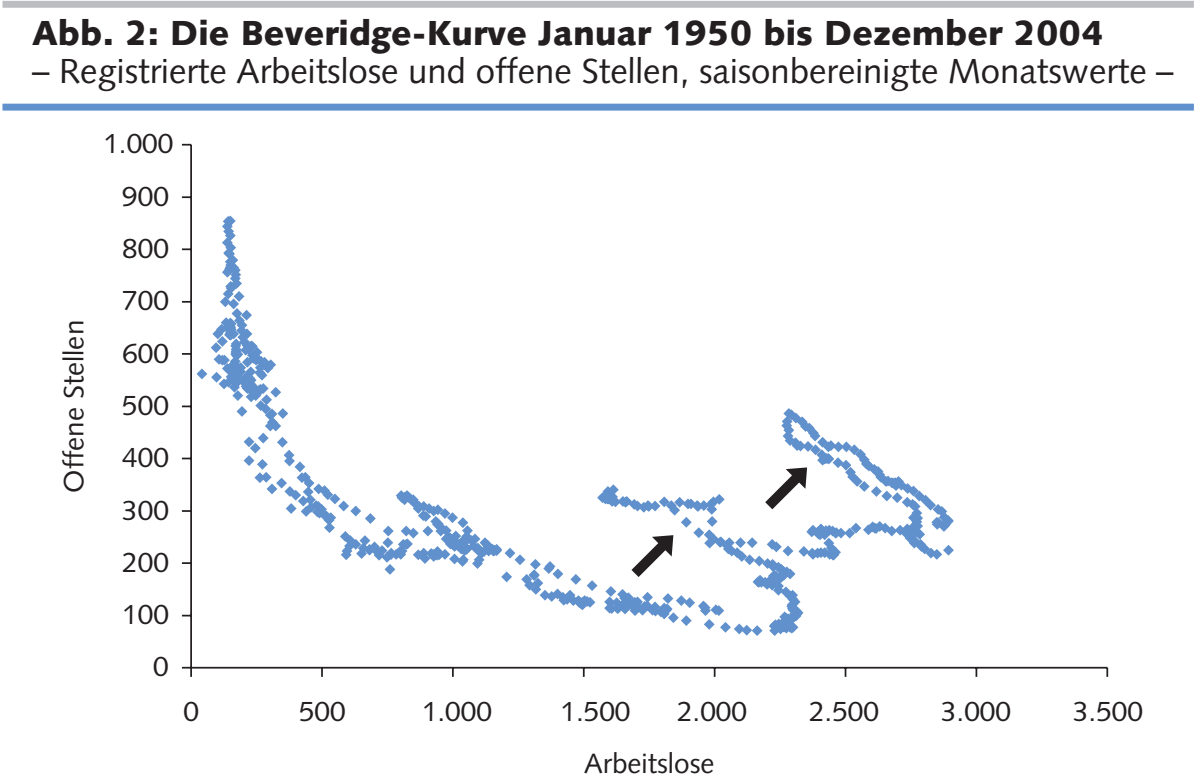

Quelle: Deutsche Bundesbank, lange Zeitreihen.

WSI MITTEILUNGEN

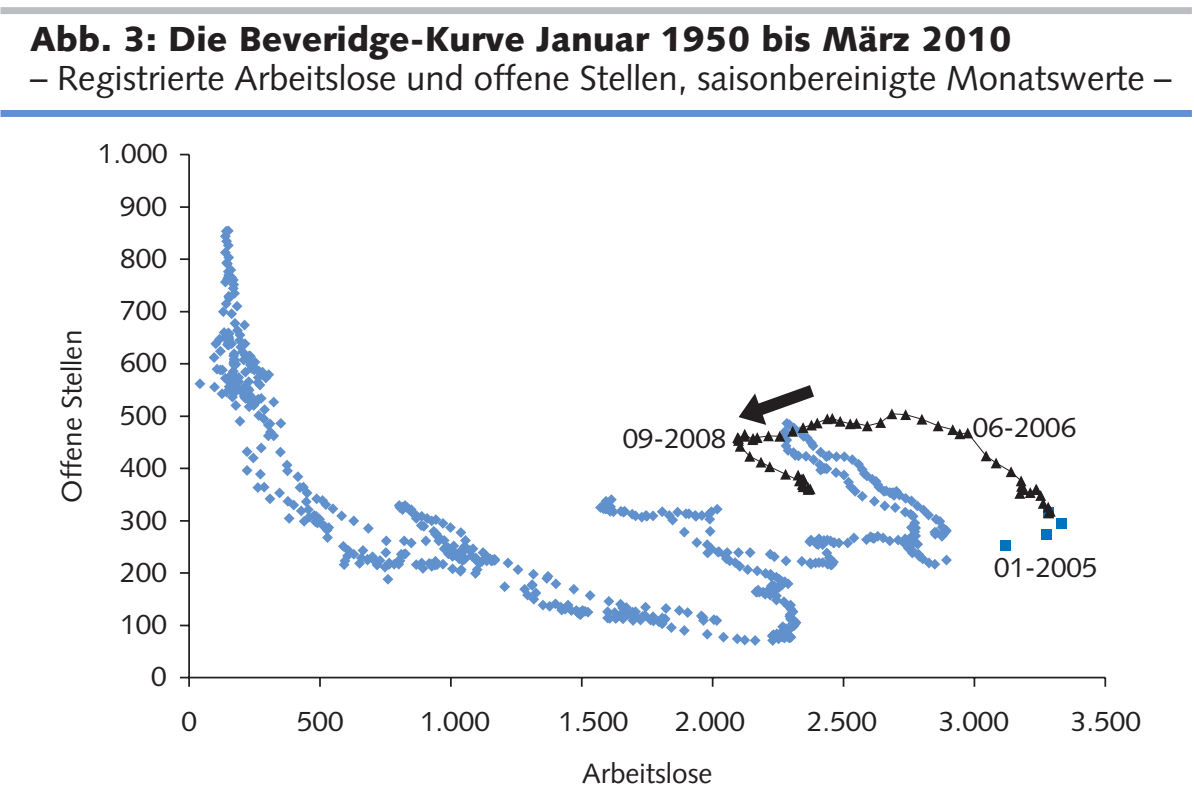

Quelle: Deutsche Bundesbank, lange Zeitreihen.

WSI MITTELLUNGEN

zum Dezember 2004. Es ist erkennbar, dass sich die Kurve in zwei Schüben nach auswärts (also vom Ursprung weg) verlagert hat. Die erste Verlagerung fand ab Mitte der 1980er Jahre statt, die zweite etwa zehn Jahre später.

Eine Auswärtsverlagerung der Beveridge-Kurve, die nicht auf eine systematische Veränderung im Erfassungsgrad zurückzuführen ist, bedeutet aber eine Verschlechterung in der Funktionsweise des Arbeitsmarktes. Abbildung 3 umfasst nun auch den Zeitraum bis zum aktuellen Rand. Zu Beginn des Jahres 2005 hat es zunächst eine weitere Auswärtsverschiebung gegeben, die auf die Veränderung in der statistischen Erfassung der erwerbsfähigen Hilfebedürftigen zurückzuführen ist. Bemerkenswert ist die Entwicklung etwa ab Mitte 2006. Die Beveridge-Kurve verschiebt sich seit diesem Zeitpunkt deutlich in Richtung Ursprung des Koordinatensystems. Dies deutet auf eine sich wieder verbessernde Funktionsfähigkeit des Arbeitsmarktes hin. Ein Beispiel: Zu Beginn

4 Da die kontrafaktische Situation („Wie sähe de deutsche Arbeitsmarkt aus, wenn es die Reformen nicht gegeben hätte?“") nicht beobachtet werden kann, sondern mithilfe aufwändiger statistischer Verfahren mit hohem Datenbedarf erschlossen werden muss, ist eine strenge Kausalanalyse derzeit allerdings noch nicht möglich. 
des Jahres 2006 gab es in Deutschland bei (saisonbereinigt) rund 500.000 registrierten offenen Stellen etwa 4,7 Mio. Arbeitslose. Bei der gleichen Zahl der offenen Stellen liegt die Arbeitslosigkeit heute bei rund 3,5 Mio. Die Veränderung ist so erheblich, dass sie nicht nur durch statistische Effekte zustande gekommen sein kann (der Erfassungsgrad der offenen Stellen müsste drastisch gesunken und die Untererfassung der Arbeitslosigkeit drastisch gestiegen sein). Unabhängig davon trägt das Argument von Niemeier (in diesem Heft) nicht, dass die Verschiebung der Beveridge-Kurve durch die Kurzarbeit zu erklären sei. Diese auffällige Verschiebung beginnt etwa ab Mitte 2006 und ist im Herbst 2008, also vor Beginn der Krise, abgeschlossen. In dieser Zeit hatte die Kurzarbeit so gut wie keine Bedeutung.

Aus meiner Sicht deutet deshalb alles darauf hin, dass sich in der Tat die Funktionsweise des Arbeitsmarktes im Zusammenspiel von guter Konjunktur und Re- formwirkungen zum Besseren gewandelt hat. Damit ist nicht gesagt, dass die Reform in jedem Detail gelungen ist und es nicht noch an vielen Stellen Nachbesserungsbedarf gibt. ${ }^{5}$ Dennoch war die Agenda 2010 unter dem Strich für den deutschen Arbeitsmarkt positiv.

5 Vgl. die Veröffentlichungen des IAB hierzu, z. B Koch, S./Kupka, P./Steinke, J. (2009): Aktivierung, Erwerbstätigkeit und Teilhabe - vier Jahre Grundsicherung für Arbeitsuchende, Bielefeld 